\title{
ON THE SPECTRUM OF NARROW PERIODIC WAVEGUIDES
}

\author{
LEONID FRIEDLANDER AND MICHAEL SOLOMYAK \\ To the memory of Leonid Romanovich Volevich
}

\begin{abstract}
This is a continuation of [1] and [2]. We consider the spectrum of the Dirichlet Laplacian in the domain $\{(x, y): 0 \leq$ $y \leq \epsilon h(x)\}$ where $h(x)$ is a positive, periodic function. The main assumption is that $h(x)$ has one point of global maximum on the period interval. We study location of the bands and prove that the band lengths are exponentially decaying as $\epsilon \rightarrow 0$.
\end{abstract}

\section{INTRODUCTION}

This paper is a continuation of the authors' works [1] and [2] where we studied the spectrum of the (positive) Laplacian $\Delta_{\epsilon}$ in a narrow strip

$$
\Omega_{\epsilon}=\{(x, y): x \in I, 0<y<\epsilon h(x)\} .
$$

There, $I$ could be either a finite interval $[-a, b]$ or the whole real line. In all cases, the Dirichlet boundary condition is imposed on the bottom $y=0$ and on the top $y=\epsilon h(x)$ of the domain $\Omega_{\epsilon}$. In the case $I=[-a, b]$, we considered both the Dirichlet (in [1]) and Neumann (in [2]) boundary conditions on the sides $x=-a$ and $x=b$ of $\Omega_{\epsilon}$. The main objective in [1] and in [2] was to understand the behavior of eigenvalues as $\epsilon \rightarrow 0$, and the main assumption was that the continuous function $h(x)$ has on $I$ a single point of global maximum. Our approach was based on a study of the behavior of the resolvent $\left(\Delta_{\epsilon}-\pi^{2} M^{-2} \epsilon^{-2}\right)^{-1}$, where $M=\max h(x)$, as $\epsilon \rightarrow 0$. Roughly speaking, we proved that this resolvent converges in norm to the operator $\mathbf{H}^{-1}$, where $\mathbf{H}$ is the operator, described below by (1.2). This requires a suitable interpretation, since the operators involved act in different Hilbert spaces. Such an interpretation was suggested in [1], [2].

In this paper, we consider the case when $h(x)$ is a periodic function. Namely, $h(x)$ is a positive, continuous $2 \pi$-periodic function on $I=$ $(-\infty, \infty)$. Let $I_{0}=[-\pi, \pi]$. We assume that

(i) $x=0$ is the only point of global maximum of $h(x)$ on $I_{0}$;

1991 Mathematics Subject Classification. 35P15. 
(ii) The function $h(x)$ is $C^{1}$ on $I_{0} \backslash\{0\}$, and in a neighborhood of $x=0$ it admits an expansion

$$
h(x)= \begin{cases}M-c_{+} x^{m}+O\left(x^{m+1}\right), & x>0, \\ M-c_{-}|x|^{m}+O\left(|x|^{m+1}\right), & x<0\end{cases}
$$

where $M, c_{ \pm}>0$ and $m \geq 1$. The spectrum of the operator $\Delta_{\epsilon}$ consists of bands $\left[a_{j}(\epsilon), b_{j}(\epsilon)\right], j=1,2, \ldots$. It is purely absolutely continuous, which was proved in [4], [5] and - under the most general assumptions about the boundary of the waveguide - in [3]. Our goal is to describe where the bands are located and to derive an upper bound for the band widths.

To formulate the main result of the paper, we need some notations (see [1], [2].) Let $\mathbf{H}$ be an operator on $L^{2}(\mathbb{R})$ given by

$$
\mathbf{H}=-\frac{d^{2}}{d x^{2}}+q(x), \quad q(x)=\left\{\begin{array}{l}
2 \pi^{2} M^{-3} c_{+} x^{m}, x>0, \\
2 \pi^{2} M^{-3} c_{-}|x|^{m}, x<0 .
\end{array}\right.
$$

Its spectrum is discrete and consists of simple eigenvalues, which we denote by $\mu_{j}$. If $m=2$ and $c_{+}=c_{-}=c$, then $\mathbf{H}$ turns into the harmonic oscillator.

Theorem 1.1. Let $h(x)$ be a continuous, positive, $2 \pi$-periodic function, and suppose that it satisfies conditions (i) and (ii). Let $\left[a_{j}(\epsilon), b_{j}(\epsilon)\right]$ be spectral bands of the operator $\Delta_{\epsilon}$ in $\Omega_{\epsilon}$ with the Dirichlet boundary conditions. Then

$$
\lim _{\epsilon \rightarrow 0} \epsilon^{2 \alpha}\left(a_{j}(\epsilon)-\frac{\pi^{2}}{M^{2} \epsilon^{2}}\right)=\mu_{j}
$$

where

$$
\alpha=2(m+2)^{-1} .
$$

Moreover, there exist a constant $\sigma>0$ and numbers $C_{j}>0$ such that

$$
b_{j}(\epsilon)-a_{j}(\epsilon) \leq C_{j} e^{-\sigma / \epsilon}
$$

The proof of the theorem is based on the Dirichlet-Neumann bracketing and on direct estimates for the difference between eigenvalues of two boundary value problems for the Laplacian. In section 2 we bound $a_{j}(\epsilon)$ and $b_{j}(\epsilon)$ by eigenvalues of axiliary problems in a bounded domain. That material is well known; we include it for the sake of completeness. The bulk of the proof of the theorem is in section 3 .

A convergence in norm of the resolvents, similar to the one proved in [1], [2], holds also in this new situation. Namely, the resolvents $\left(\Delta_{\epsilon}-\pi^{2} M^{-2} \epsilon^{-2}\right)^{-1}$ converge, in an appropriate sense, to the orthogonal 
sum of infinitely many copies of the operator $\mathbf{H}^{-1}$, see (1.2). We do not go into details, since this gives nothing for the better understanding of our main result which is the estimate (1.5).

Acknowledgments. The work was mostly done when both authors visited the Isaac Newton Institute for Mathematical Sciences in Cambridge, UK. We acknowledge the hospitality of the Newton Institute. The first author was partially supported by the NSF grant DMS 0648786 .

\section{Dirichlet-Neumann BRACKEting}

The Floquet theory tells us that the spectrum of the operator $\Delta_{\epsilon}$ is the union of the spectra of operators $\Delta_{\epsilon, k}, 0 \leq k<1$; these are the Laplacians in $\Omega_{\epsilon}$ with the domain

$$
\left\{u \in H^{2}\left(\Omega_{\epsilon}\right) \cap H_{0}^{1}\left(\Omega_{\epsilon}\right): u(x, y+2 \pi)=e^{i k} u(x, y)\right\} .
$$

Let $\Omega_{\epsilon}^{0}=\{(x, y):-\pi \leq x \leq \pi, 0 \leq y \leq \epsilon h(x)\}$ be the fundamental domain of the $\mathbb{Z}$-action $(x, y) \mapsto(x+2 \pi n, y), n \in \mathbb{Z}$, on $\Omega_{\epsilon}$. The operator $\Delta_{\epsilon, k}$ can be considered as an operator in $\Omega_{\epsilon}^{0}$; it is generated by the quadratic form

$$
Q(u)=\int_{\Omega_{\epsilon}^{0}}|\nabla u|^{2} d x d u
$$

with the domain

$\mathfrak{d}_{\epsilon, k}=\left\{u \in H^{1}\left(\Omega_{\epsilon}^{0}\right): u(x, 0)=u(x, \epsilon h(x))=0, u(\pi, y)=e^{i k} u(-\pi, y)\right\}$.

The operator $\Delta_{\epsilon, k}$ has discrete spectrum. Let

$$
0<\nu_{1}(\epsilon, k) \leq \nu_{2}(\epsilon, k) \leq \cdots
$$

be its eigenvalues.

By $\Delta_{\epsilon, D N}$ and $\Delta_{\epsilon, D}$ we denote the Laplacians in $\Omega_{\epsilon}^{0}$, with the Dirichlet condition imposed on the bottom and on the top of $\Omega_{\epsilon}^{0}$; the conditions on the sides $x= \pm \pi$ are Neumann and Dirichlet, respectively. These operators are generated by the same quadratic form (2.1), but now it is defined on the domains

$\mathfrak{d}_{\epsilon, D N}=\left\{u \in H^{1}\left(\Omega_{\epsilon}^{0}\right): u(x, 0)=u(x, \epsilon h(x))=0\right\}$ and $\mathfrak{d}_{\epsilon, N}=H_{0}^{1}\left(\Omega_{\epsilon}^{0}\right)$.

Let $\lambda_{j}(\epsilon, D N)$ and $\lambda_{j}(\epsilon, D)$ be the eigenvalues of the operators $\Delta_{\epsilon, D N}$ and $\Delta_{\epsilon, D}$, respectively. The inclusions $\mathfrak{d}_{\epsilon, D} \subset \mathfrak{d}_{\epsilon, k} \subset \mathfrak{d}_{\epsilon, D N}$ (see (2.2) and (2.3)) imply

$$
\lambda_{j}(\epsilon, D N) \leq \nu_{j}(\epsilon, k) \leq \lambda_{j}(\epsilon, D) .
$$


Theorem 2.1 from [2] says that both $\lambda_{j}(\epsilon, D N)$ and $\lambda_{j}(\epsilon, D)$ satisfy

$$
\lim _{\epsilon \rightarrow 0} \epsilon^{2 \alpha}\left(\lambda_{j}(\epsilon)-\frac{\pi^{2}}{M^{2} \epsilon^{2}}\right)=\mu_{j}
$$

Therefore, for every $n$, first $n+1$ eigenvalues of $\Delta_{\epsilon, D N}$ and $\Delta_{\epsilon, D}$ interlace

$$
\begin{gathered}
\lambda_{1}(\epsilon, D N) \leq \lambda_{1}(\epsilon, D)<\lambda_{2}(\epsilon, D N) \leq \lambda_{2}(\epsilon, D)<\cdots \\
<\lambda_{n}(\epsilon, D N) \leq \lambda_{n}(\epsilon, D)<\lambda_{n+1}(\epsilon, D N)
\end{gathered}
$$

when $\epsilon$ is small enough, $\epsilon<\epsilon_{0}(n)$ (actually, in the last formula, one can replace all inequalities $\leq$ by $<$; it follows from the formula (2.6) below.) Then, the spectral bands $\left[a_{j}(\epsilon), b_{j}(\epsilon)\right]$ lie between $\lambda_{j}(\epsilon, D N)$ and $\lambda_{j}(\epsilon, D)$ when $j \leq n$,

$$
\lambda_{j}(\epsilon, D N) \leq a_{j}(\epsilon)<b_{j}(\epsilon) \leq \lambda_{j}(\epsilon, D) .
$$

The two-term asymptotics (1.3) for $a_{j}(\epsilon)$ follows from (2.5) and (2.6). Notice that (1.3) holds if one replaces $a_{j}(\epsilon)$ by $b_{j}(\epsilon)$. In addition, $(2.6)$ implies

$$
b_{j}(\epsilon)-a_{j}(\epsilon) \leq \lambda_{j}(\epsilon, D)-\lambda_{j}(\epsilon, D N) \text {. }
$$

3. The estimate FoR $\lambda_{j}(\epsilon, D)-\lambda_{j}(\epsilon, D N)$

In this section, we will prove that there exists a constant $\sigma$ such that

$$
\lambda_{j}(\epsilon, D)-\lambda_{j}(\epsilon, D N) \leq C_{j} e^{-\sigma / \epsilon}
$$

for some $C_{j}$. The estimate (1.5) follow from (2.6) and (3.1). The operators $\Delta_{\epsilon, D N}$ and $\Delta_{\epsilon, D}$ are unitary equivalent to the operators $\mathfrak{L}_{\epsilon, D N}$ and $\mathfrak{L}_{\epsilon, D}$, respectively, that are given by the differential expression

$$
L_{\epsilon}=-\frac{\partial^{2}}{\partial x^{2}}-\frac{1}{\epsilon^{2}} \frac{\partial^{2}}{\partial y^{2}}
$$

in

$$
\Omega=\{(x, y):-\pi \leq x \leq \pi, 0 \leq y \leq h(x)\} ;
$$

the boundary conditions are always Dirichlet on the top and on the bottom of $\Omega$. For the operator $\mathfrak{L}_{\epsilon, D N}$, one takes the Neumann conditions at the sides $x= \pm \pi$; for the operator $\mathfrak{L}_{\epsilon, D}$ one takes the Dirichlet conditions on the sides as well. We recall that function $h$ has the only point of global maximum on $[-\pi, \pi]$, and $h(0)=M$. First, we will show that eigenfunctions of the operator $\mathfrak{L}_{\epsilon, D N}$ are very small near the right and left sides of $\Omega$. Let

$$
m=\max \left\{h(x): \frac{\pi}{3} \leq|x| \leq \pi\right\},
$$


and let $u(x, y)$ be an eigenfunction of $\mathfrak{L}_{\epsilon, D N}$ :

$$
u_{x x}+\frac{1}{\epsilon^{2}} u_{y y}+\frac{\mu}{\epsilon^{2}} u=0, u(x, 0)=u(x, h(x))=0, u_{x}( \pm \pi, y)=0,
$$

with

$$
\mu \leq \frac{\pi^{2}}{m^{2}}-\delta, \quad \delta>0
$$

We will show that there exist constants $C$ and $\eta$ that depend on $\delta$ only such that

$$
\int_{0}^{h(x)} u(x, y)^{2} d y \leq C e^{-\eta / \epsilon}\|u\|_{L^{2}(\Omega)}^{2}
$$

when $\pi / 2 \leq|x| \leq \pi$. To prove (3.2) one can assume $\|u\|_{L^{2}(\Omega)}=1$. We will take $-\pi \leq x \leq-\pi / 2$; the case $\pi / 2 \leq x \leq \pi$ is similar. Let

$$
F(x)=\int_{0}^{h(x)} u(x, y)^{2} d y .
$$

Let $-\pi \leq x<z \leq-\pi / 3$. We multiply (3.4) by $u(x, y)$ and integrate the resulting equality over

$$
\Pi_{x, z}=\{(\xi, y): x \leq \xi \leq z, 0 \leq y \leq h(\xi)\}
$$

to get

$$
\begin{gathered}
\int_{\Pi_{x, z}} u_{x}^{2}(\xi, y) d \xi d y+\int_{0}^{h(z)} u_{x}(z, y) u(z, y) d y-\int_{0}^{h(x)} u_{x}(x, y) u(x, y) d y \\
-\frac{1}{\epsilon^{2}} \int_{\Pi_{x, z}} u_{y}^{2}(\xi, y) d \xi d y+\frac{\mu}{\epsilon^{2}} \int_{\Pi_{x, z}} u^{2}(\xi, y) d \xi d y=0 .
\end{gathered}
$$

Notice that the second term in the last equality equals $F^{\prime}(z) / 2$, the third term equals $F^{\prime}(x) / 2$, and the last term equals $\epsilon^{-2} \mu \int_{x}^{z} F(\xi) d \xi$, so it can be re-written as

$$
\begin{aligned}
\frac{F^{\prime}(z)-F^{\prime}(x)}{2}=\frac{1}{\epsilon^{2}} & \int_{\Pi_{x, z}} u_{y}^{2}(\xi, y) d \xi d y-\frac{\mu}{\epsilon^{2}} \int_{x}^{z} F(\xi) d \xi+ \\
& \int_{\Pi_{x, z}} u_{x}^{2}(\xi, y) d \xi d y .
\end{aligned}
$$

The estimate $h(\xi) \leq m$ for $x \leq \xi \leq z$ implies

$$
\int_{0}^{h(\xi)} u_{y}^{2}(\xi, y) d y \geq \frac{\pi^{2}}{m^{2}} F(\xi),
$$

and

$$
\int_{\Pi_{x, z}} u_{y}^{2}(\xi, y) d \xi d y \geq \frac{\pi^{2}}{m^{2}} \int_{x}^{z} F(\xi) d \xi
$$


The last inequality and (3.3) imply

$$
\frac{F^{\prime}(z)-F^{\prime}(x)}{2} \geq \frac{\delta}{\epsilon^{2}} \int_{x}^{z} F(\xi) d \xi
$$

We divide (3.6) by $z-x$ and take the limit $z \rightarrow x$ to get

$$
F^{\prime \prime}(x) \geq \frac{2 \delta}{\epsilon^{2}} F(x), \quad|x| \geq \frac{\pi}{3} .
$$

Notice that $F^{\prime}(-\pi)=0$. Therefore $F^{\prime}(x) \geq 0$ when $-\pi \leq x \leq-\pi / 3$; together with (3.7) this implies

$$
F(z) \geq F(x) \cosh \left(\frac{\sqrt{2 \delta}}{\epsilon}(z-x)\right), \quad x \leq z \leq-\frac{\pi}{3} .
$$

Let $-\pi \leq x \leq-\pi / 2$. It follows from (3.8) that

$$
F(z) \geq \frac{1}{2} F(x) e^{\sqrt{2 a \delta} /(12 \epsilon)}, \quad-\frac{5 \pi}{12} \leq z \leq-\frac{\pi}{3} .
$$

Therefore,

$$
1 \geq \int_{-5 \pi / 12}^{-\pi / 3} F(z) d z \geq \frac{\pi}{24} F(x) e^{\sqrt{2 \pi \delta} /(12 \epsilon)},
$$

and

$$
F(x) \leq \frac{24}{\pi} e^{-\sqrt{2 \pi \delta} /(12 \epsilon)} .
$$

One sets $C=24 / \pi$ and $\eta=\pi \sqrt{2 \delta} / 12$ to get (3.4).

We are ready now to prove (3.1). Let $\left\{u_{l}(x, y)\right\}$ be an ortho-normal basis composed by eigenfunctions of the operator $\mathfrak{L}_{\epsilon, D N}$,

$$
L_{\epsilon} u_{l}(x, y)=\lambda_{l, D N}(\epsilon) u_{l}(x, y) .
$$

By $\mathcal{L}_{j}$ we denote the span of $u_{1}(x, y), \ldots, u_{j}(x, y)$. Let $\chi(x) \in C_{0}^{\infty}(-\pi, \pi)$ be a cut-off function such that $0 \leq \chi(x) \leq 1$ and $\chi(x)=1$ when $|x| \leq \pi / 2$. Let $\mathcal{M}_{j}=\chi(x) \mathcal{L}_{j} \subset \overline{H_{0}^{1}}(\Omega)$. Then $\operatorname{dim} \mathcal{M}_{j}=j$. Take a function $v(x, y)=\chi(x) u(x, y) \in \mathcal{M}_{j}$. Then an upper bound for the ration

$$
\int_{\Omega}\left(v_{x}^{2}+\frac{1}{\epsilon^{2}} v_{y}^{2}\right) d x d y / \int_{\Omega} v^{2} d x d y
$$

will be an upper bound for $\lambda_{j, D}(\epsilon)$. One can assume that $\int_{\Omega} u^{2} d x d y=1$. Then

$$
u(x, y)=\sum_{l=1}^{j} c_{l} u_{l}(x, y), \quad \sum_{l=1}^{j} c_{l}^{2}=1
$$

and

$$
\int_{\Omega} v^{2} d x d y \geq 1-\int_{\Omega \cap\{\pi / 2 \leq|x| \leq \pi\}} u^{2} d x d y
$$


Take any number $\delta, 0<\delta<\pi^{2}\left(m^{-2}-M^{-2}\right)$ (here $m$ is the same as in (3.3).) Theorem 2.1 from [2] implies $\lim _{\epsilon \rightarrow 0} \epsilon^{2} \lambda_{j, D}(\epsilon)=\pi^{2} / M^{2}$; therefore, for $\epsilon$ small enough,

$$
\lambda_{j, D}(\epsilon) \leq \frac{1}{\epsilon^{2}}\left(\frac{\pi^{2}}{m^{2}}-\delta\right) .
$$

One has $\lambda_{l, D N}(\epsilon) \leq \lambda_{j, D}(\epsilon)$ for $l \leq j$, so (3.4) implies

$$
\int_{\Omega} v^{2} d x d y \geq 1-C_{j}^{\prime} e^{-\eta / \epsilon}
$$

Let us now get an estimate for the numerator in (3.9):

$$
\begin{aligned}
& \int_{\Omega}\left(v_{x}^{2}+\frac{1}{\epsilon^{2}} v_{y}^{2}\right) d x d y=\int_{\Omega}\left(\chi^{2}(x)\left(u_{x}^{2}+\frac{1}{\epsilon^{2}} u_{y}^{2}\right) d x d y\right. \\
& \quad+\int_{\Omega} \chi^{\prime}(x)^{2} u^{2} d x d y+2 \int_{\Omega} \chi(x) \chi^{\prime}(x) u u_{x} d x d y \\
& \leq \lambda_{j, D N}(\epsilon)-\int_{\Omega} \chi(x) \chi^{\prime \prime}(x) u^{2} d x d y \\
& \leq \lambda_{j, D N}(\epsilon)+C^{\prime} \int_{\Omega \cap\{\pi / 2 \leq|x| \leq \pi\}} u^{2} d x d y \leq \lambda_{j, D N}(\epsilon)+C_{j}^{\prime \prime} e^{-\eta / \epsilon}
\end{aligned}
$$

Inequalities (3.10) and (3.11) imply that the quotient (3.9) does not exceed

Therefore,

$$
\frac{\mu_{j}(\epsilon)+C_{j}^{\prime \prime} e^{-\eta / \epsilon}}{1-C_{j}^{\prime} e^{-\eta / \epsilon}} \leq \lambda_{j, D N}(\epsilon)+C_{j} e^{-\eta / \epsilon} .
$$

$$
\lambda_{j, D}(\epsilon) \leq \lambda_{j, D N}(\epsilon)+C_{j} e^{-\eta / \epsilon}
$$

\section{REFERENCES}

[1] L. Friedlander, M. Solomyak, On the spectrum of the Dirichlet Laplacian in a narrow strip, Israeli Math. J., to appear.

[2] L. Friedlander, M. Solomyak, On the spectrum of the Dirichlet Laplacian in a narrow strip II, preprint arXiv:0710.1886

[3] R. Shterenberg, Absolute continuity of the spectrum of two-dimensional periodic Schrödinger operator with strongly subordinate magnetic potential (Russian), Zap. Nauchn. Sem. S.-Peterburg. Otdel. Mat. Inst. Steklov. (POMI) 303 (2003), Issled. po Linein. Oper. i Teor. Funkts. 31, 279-320, 325-326; translation in J. Math. Sci. (N. Y.) 129 (2005), no. 4, 4087-4109.

[4] A. Sobolev, J. Walthoe, Absolute continuity in periodic waveguides, Proc. London Math. Soc., 85 (2002), 717-741

[5] T. Suslina, R. Shterenberg, Absolute continuity of the spectrum of the magnetic Schrödinger operator with a metric in a two-dimensional periodic waveguide (Russian), Algebra i Analiz 14 (2002), no. 2, 159-206; translation in St. Petersburg Math. J. 14 (2003), no. 2, 305-343. 
Department of Mathematics, University of Arizona, Tucson, AriZONA, USA

E-mail address: friedlan@math.arizona.edu

Department of Mathematics, The Weizmann Institute of Science, REHOVOT 76100, ISRAEL

E-mail address: michail.solomyak@wisdom.weizmann.ac.il 